Impact ionization in GaAs: A screened exchange density-functional approach

\title{
S. Picozzi
}

INFM - Dip. Fisica, Univ. L'Aquila, 67010 Coppito (L'Aquila), Italy

R. Asahi

Toyota Central R \& D Labs, Inc., Nagakute, Japan

C. B. Geller

Bechtel Bettis, Inc., West Mifflin, PA 15122-0079

A. Continenza

NFM - Dip. Fisica, Univ. L'Aquila, 67010 Coppito (L'Aquila), Italy

A. J. Freeman

Depart. of Physics and Astronomy and Materials Research Center, Northwestern Univ., Evanston, IL 60208

\section{NOTICE}

This report was prepared as an account of work sponsored by an agency of the United States Government. Neither the United States Government nor any agency thereof, nor any of their employees, nor any of their contractors, subcontractors or their employees, makes any warranty, express or implied, or assumes any legal liability or responsibility for the accuracy, completeness, or any third party's use or the results of such use of any information, apparatus, product, or process disclosed, or represents that its use would not infringe privately owned rights. Reference herein to any specific commercial product, process, or service by trade name, trademark, manufacturer, or otherwise, does not necessarily constitute or imply its endorsement, recommendation, or favoring by the United States Government or any agency thereof or its contractors or subcontractors. The views and opinions of authors expressed herein do not necessarily state or reflect those of the United States Government or any agency thereof. 
Impact ionization in GaAs: A screened exchange density-functional approach

\section{S. Picozzi}

INFM - Dip. Fisica, Univ. L'Aquila, 67010 Coppito (L'Aquila), Italy

R. Asahi

Toyota Central R \& D Labs, Inc., Nagakute, Japan

C. B. Geller

Bettis Atomic Power Laboratory, West Mifflin, PA 15122-0079

A. Continenza

NFM - Dip. Fisica, Univ. L'Aquila, 67010 Coppito (L'Aquila), Italy

A. J. Freeman

Depart. of Physics and Astronomy and Materials Research Center, Northwestern Univ., Evanston, IL 60208

\section{NOTICE}

This report was prepared as an account of work sponsored by an agency of the United States Government. Neither the United States Government nor any agency thereof, nor any of their employees, nor any of their contractors, subcontractors or their employees, makes any warranty, express or implied, or assumes any legal liability or responsibility for the accuracy, completeness, or any third party's use or the results of such use of any information, apparatus, product, or process disclosed, or represents that its use would not infringe privately owned rights. Reference herein to any specific commercial product, process, or service by trade name, trademark, manufacturer, or otherwise, does not necessarily constitute or imply its endorsement, recommendation, or favoring by the United States Government or any agency thereof or its contractors or subcontractors. The views and opinions of authors expressed herein do not necessarily state or reflect those of the United States Government or any agency thereof. 


\title{
Impact ionization in GaAs: A screened exchange density-functional approach
}

\author{
S. Picozzi* \\ INFM - Dip. Fisica, Univ. L'Aquila, 67010 Coppito (L'Aquila), Italy \\ R. Asahi \\ Toyota Central R \& D Labs., Inc., Nagakute, Japan \\ C.B. Geller \\ Bettis Atomic Power Laboratory, West Mifflin, Pennsylvania 15122 \\ A. Continenza \\ INFM -Dip. Fisica, Univ. L'Aquila, 67010 Coppito (L'Aquila), Italy
}

A. J. Freeman

Department of Physics and Astronomy and Materials Research Center, Northwestern University, Evanston, Illinois 60208

(Received 23 August 2001; revised manuscript received 6 December 2001; published 28 February 2002)

\begin{abstract}
Results are presented of a fully ab initio calculation of impact ionization rates in GaAs within the density functional theory framework, using a screened-exchange formalism and the highly precise all-electron fullpotential linearized augmented plane wave method. The calculated impact ionization rates show a marked orientation dependence in $\mathbf{k}$ space, indicating the strong restrictions imposed by the conservation of energy and momentum. This anisotropy diminishes as the impacting electron energy increases. A Keldysh type fit performed on the energy-dependent rate shows a rather soft edge and a threshold energy greater than the direct band gap. The consistency with available Monte Carlo and empirical pseudopotential calculations shows the reliability of our approach and paves the way to ab initio calculations of pair production rates in new and more complex materials.
\end{abstract}

DOI: 10.1103/PhysRevB.65.113206

PACS number(s): 79.20.Ap, 71.15.Mb

Band-to-band impact ionization (II) is a carrier-carrier scattering process in which an energetic carrier creates an electron-hole pair through the excitation of a valence electron $\left(e^{-}\right)$in the conduction band. ${ }^{1,2}$ This process is fundamental in small high-speed devices, both as a charge multiplication (e.g., avalanche photodiodes) and as a detrimental mechanism (e.g., field effect transistors). We present a fully first-principles approach based on screened-exchange ${ }^{3,4}$ density functional theory (DFT) (Ref. 5) that allows a full understanding of the basic mechanisms and physical quantities affecting the II process. Our final goal is to tune the rates according to technological requirements through band structure "engineering" for simple and complex materials, without need of ad hoc parameters or pseudoatoms.

All previous theoretical treatments have employed approximate band structures and matrix elements, based on $\mathbf{k} \cdot \mathbf{p}$, Monte Carlo (MC) (Refs. 6-8) or empirical pseudopotential (EPP) (Refs. 9-11) formalisms. To our knowledge, the work herein reported is the first fully ab initio calculation of II rates. We selected the most studied direct gap semiconductor GaAs since a variety of II results obtained through different approaches is available for GaAs and so multiple comparisons can be made with results of the current first principles method.

We consider an $e^{-}$-initiated II process (shown schematically in Fig. 1). According to Fermi's Golden rule, the rate $r\left(n_{1}, \mathbf{k}_{1}\right)$ at which the impacting $e^{-}$in a $\left(n_{1}, \mathbf{k}_{1}\right)$ state can produce II is obtained as

$$
\begin{aligned}
r\left(n_{1}, \mathbf{k}_{1}\right)= & 2 \frac{2 \pi}{\hbar} \sum_{n_{2}, n_{3}, n_{4}} \int d^{3} \mathbf{k}_{2} \int d^{3} \mathbf{k}_{3}|M|^{2} \delta\left(E_{\mathbf{k}_{1}}^{n_{1}}+E_{\mathbf{k}_{2}}^{n_{2}}\right. \\
& \left.-E_{\mathbf{k}_{3}}^{n_{3}}-E_{\mathbf{k}_{1}+\mathbf{k}_{2}-\mathbf{k}_{3}}^{n_{4}}\right)
\end{aligned}
$$

where the band indices $n_{3}, n_{4}\left(n_{2}\right)$ run over the conduction (valence) states and $\mathbf{k}_{1}, \mathbf{k}_{2}, \mathbf{k}_{3}$ are $\mathbf{k}$ points in the full Brillouin zone (BZ); $\delta\left(E_{\mathbf{k}_{1}}^{n_{1}}+E_{\mathbf{k}_{2}}^{n_{2}}-E_{\mathbf{k}_{3}}^{n_{3}}-E_{\mathbf{k}_{1}+\mathbf{k}_{2}-\mathbf{k}_{3}}^{n_{4}}\right)$ shows the energy conservation. The antisymmetrized screened Coulomb matrix element is obtained by adding the probabilities in the singlet and triplet states $|M|^{2}=\frac{1}{4}\left(\left|M_{D}+M_{E}\right|^{2}\right.$ $+3\left|M_{D}-M_{E}\right|^{2}$ ) where $M_{D}$ and $M_{E}$ are direct and exchange

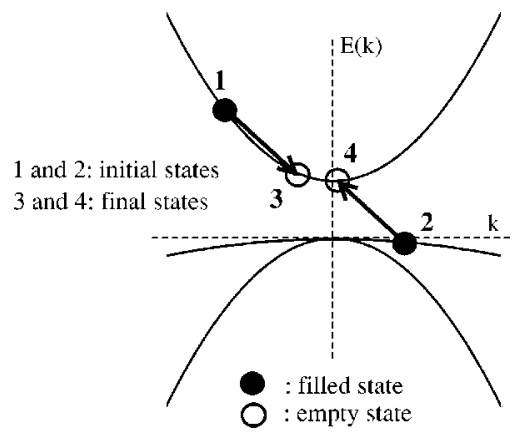

FIG. 1. Electron-initiated II process. The initial electrons in the conduction and valence bands are in states 1 and 2, respectively; after the transition, the final electrons in the conduction bands are in states 3 and 4 . 
(obtained from the direct term by exchanging final states) matrix elements. Each direct matrix element is expressed as

$$
\begin{aligned}
M_{D}= & \frac{4 \pi e^{2}}{\Omega} \sum_{\mathbf{G}_{0}, \mathbf{G}_{\mathbf{U}}} \delta\left(\mathbf{k}_{1}+\mathbf{k}_{2}-\mathbf{k}_{3}-\mathbf{k}_{4}\right. \\
& \left.+\mathbf{G}_{0}\right) \frac{\rho_{n_{3}, \mathbf{k}_{3} ; n_{1}, \mathbf{k}_{1}}\left(\mathbf{G}_{\mathbf{U}}\right) \rho_{n_{4}, \mathbf{k}_{4} ; n_{2}, \mathbf{k}_{2}}\left(\mathbf{G}_{0}-\mathbf{G}_{\mathbf{U}}\right)}{\varepsilon(q)\left(\left|\mathbf{k}_{1}-\mathbf{k}_{3}+\mathbf{G}_{\mathbf{U}}\right|^{2}+\lambda^{2}\right)},
\end{aligned}
$$

where the $\delta$ function shows the momentum (k) conservation and $\rho_{n_{f}, \mathbf{k}_{f} ; n_{i}, \mathbf{k}_{i}}(\mathbf{G})$ is the Fourier transform of the overlap matrix of the wave functions $\rho_{n_{f}, \mathbf{k}_{f} ; n_{i}, \mathbf{k}_{i}}(\mathbf{r})$ $=\Psi_{n_{f}, \mathbf{k}_{f}}^{*}(\mathbf{r}) \Psi_{n_{i}, \mathbf{k}_{i}}(\mathbf{r})$. The subscripts $i$ and $f$ denote initial and final states; $e$ is the electron charge; $\Omega$ is the unit cell volume; $q=\left|\mathbf{k}_{1}-\mathbf{k}_{3}+\mathbf{G}_{\mathbf{U}}\right|$ is the momentum transfer, and $\mathbf{G}_{0}, \mathbf{G}_{\mathbf{U}}$ are reciprocal lattice vectors.

The interaction between valence and conduction electrons is modeled by a Coulomb potential screened through a $q$-dependent static model dielectric function ${ }^{12} \varepsilon(q)$ which is particularly accurate for semiconductors. The interaction between conduction electrons is modeled through a Debye potential ${ }^{2}$ both the temperature $T$ and the carrier density in the conduction band $n_{0}$ are taken into account through an inverse Debye screening length ${ }^{9}$ given by $\lambda$ $=\sqrt{4 \pi n_{0} e^{2} / K_{B} T}$, where $K_{B}$ is the Boltzmann constant. Here, we used $T=300 \mathrm{~K}$ and $n_{0}=1 \times 10^{16} \mathrm{~cm}^{-3}$. To overcome the well-known shortcomings of the local density approximation (LDA) to DFT when dealing with excited states, ${ }^{5}$ we performed self-consistent screened exchange LDA (sX-LDA) $)^{3,4,13,14}$ calculations, as implemented ${ }^{13}$ within the highly precise full-potential linearized augmented plane wave (FLAPW) (Ref. 15) method. The sX-LDA singleparticle equations are expressed as

$$
\begin{aligned}
& \hat{h}^{\mathrm{LDA}} \Psi_{n, \mathbf{k}}(\mathbf{r})+\int d \mathbf{r}^{\prime} v_{s x}^{\mathrm{NL}}\left(\mathbf{r}, \mathbf{r}^{\prime}\right) \Psi_{n, \mathbf{k}}\left(\mathbf{r}^{\prime}\right)-v_{s x}^{L} \Psi_{n, \mathbf{k}}(\mathbf{r}) \\
& \quad=E_{\mathbf{k}}^{n} \Psi_{n, \mathbf{k}}(\mathbf{r}),
\end{aligned}
$$

where $\hat{h}^{\mathrm{LDA}}$ is the usual LDA Hamiltonian, ${ }^{5} v_{s x}^{L}$ is a local screened exchange potential ${ }^{4,13}$ and the nonlocal ThomasFermi sX operator (using a set of special $k$ points $\mathbf{q}_{s}{ }^{16}$ ) is

$$
v_{s x}^{\mathrm{NL}}\left(\mathbf{r}, \mathbf{r}^{\prime}\right)=-\sum_{n_{j}^{\text {occ }}} \sum_{\mathbf{q}_{s}} \frac{\Psi_{n_{j, \mathbf{q}_{s}}}(\mathbf{r}) e^{-k_{T F}\left|\mathbf{r}-\mathbf{r}^{\prime}\right|} \Psi_{n_{j, \mathbf{q}_{s}}^{*}}^{*}\left(\mathbf{r}^{\prime}\right)}{\left|\mathbf{r}-\mathbf{r}^{\prime}\right|},
$$

$k_{\mathrm{TF}}$ being the Thomas Fermi wave vector. The sX-LDA matrix elements ${ }^{13}$ are constructed in a way similar to a HartreeFock calculation. ${ }^{17}$ After diagonalization, the newly obtained eigenfunctions were used to update the screened Fock exchange operator in the following iteration until the charge densities and wave functions satisfied self consistency. During the initial LDA (and the following sX-LDA) selfconsistent iterations, we used a cut off equal to 9 Ry (6 Ry) in the wave vectors and $l \leqslant 8(l \leqslant 4)$ inside the muffin-tin spheres chosen as $R_{\mathrm{MT}}^{\mathrm{Ga}}=R_{\mathrm{MT}}^{\mathrm{As}}=2.3$ a.u. The summation over the irreducible $\mathrm{BZ}$ was done using two special $\mathbf{k}$ points. ${ }^{16}$
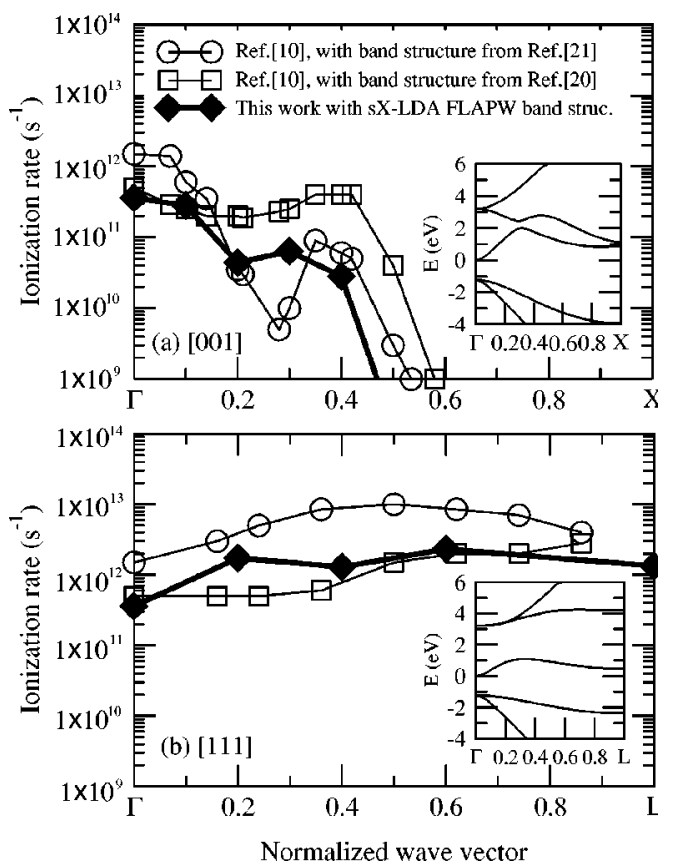

FIG. 2. Calculated ionization rates (in $\mathrm{s}^{-1}$ ) for impacting $e^{-}$in the second conduction band and wave vector along (a) $\Gamma-X$ and (b) $\Gamma-L$, vs normalized wave vector (filled diamonds). The open symbols show previous results from Ref. 10. In each panel, the inset shows the band structure along the corresponding symmetry lines [the zero of energy is set to the conduction band minimum (CBM)].

The sX-LDA approach can be recast ${ }^{4}$ within a generalized DFT formalism in which the inclusion of a nonlocal sX functional highly improves the description of the conduction band states compared with a bare LDA approach. This is essential in the present context, since the transitions considered always involve conduction states. The many-fold integration over the full $\mathrm{BZ}$ was carried out using the technique proposed by Sano and Yoshii, ${ }^{9}$ based on a regular grid of $\mathbf{k}$ points [with an interval length $\Delta k=(1 / n) 2 \pi / a, n=10]$. Similarly to band-structure calculations in the sX-LDA FLAPW method, ${ }^{13}$ the sX-LDA self-consistent charge density and sX operator [Eq. (4)] were used to evaluate the sX-LDA eigenenergies and eigenfunctions on the SanoYoshii grid. The energy $\delta$ function ${ }^{10,9}$ is approximated by a "top-hat" function, i.e., as a rectangle $\Delta_{E}$ wide and $1 / \Delta_{E}$ high (we used $\Delta_{E}=0.2 \mathrm{eV}^{10}$ ). Spin-orbit coupling was neglected; Umklapp processes for $\mathbf{G}_{\mathbf{U}} \neq 0$ were fully included in the formalism, whereas $\mathbf{G}_{0} \neq 0$ Umklapp terms were neglected. ${ }^{18}$ We also have not considered: (i) phonon-assisted transitions, that would relax the $\mathbf{k}$ conservation requirements among the four involved electronic states (i.e., k conservation would be satisfied through phonon participation) and (ii) the influence of the electric field on the collision term, i.e., the "intracollisional field effect." 19

The results for II rates initiated by electrons in the second lowest conduction band with wave vectors along [001] and [111] are shown in Fig. 2. The insets show the band structure along the same symmetry lines as in the main panels. Due to energy and $\mathbf{k}$ conservation constraints, there is a marked $\mathbf{k}$-space anisotropy in the II rate that is orders of magnitude 


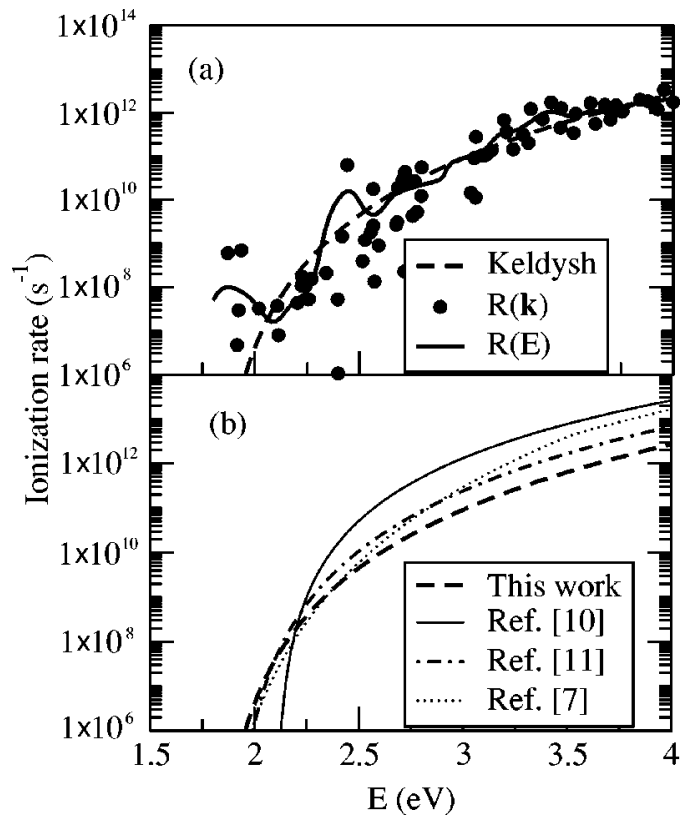

FIG. 3. (a) Ionization rates (in $\mathrm{s}^{-1}$ ) for initial $e^{-}$in the three lowest conduction bands vs their energies (the zero of energy is set to the CBM). Filled circles: wave vector dependent ionization rate $R(\mathbf{k})$; solid line: energy dependent ionization rate $R(E)$; dashed line: Keldysh fit. (b) Keldysh-type fits taken from this work (dashed bold line), Ref. 10 (thin solid line), Ref. 11 (dot-dashed line), and Ref. 7 (dotted line).

higher along [111] ( $\Gamma$ to $L$ ) than along [001] $(\Gamma$ to $X)$. Note that the second lowest conduction band in GaAs shows a decrease in energy from $\Gamma$ towards $X$, but increases with increasing $|\mathbf{k}|$ in other directions, such as [111]. We therefore expect, and find [see Fig. 2(a)], a "wave-vector antithreshold" along the [001] axis at which II no longer becomes possible. Our calculated points are compared with those of other calculations ${ }^{10}$ obtained with EPP that investigated the effect on the predicted rate assuming two different band structures. ${ }^{20,21}$ This comparison makes clear the importance of employing an accurate band structure (such as sX-LDA FLAPW), to obtain reliable rates. The degree of agreement between these calculations and ours is reasonable, given that the band structure and some of the numerical approximations ${ }^{10}$ made in the evaluation of matrix elements differ.

The calculated II rates for GaAs are shown in Fig. 3(a) for processes initiated by electrons in the three lowest conduction bands vs their impacting energy. The scattered points in the low-energy region reflect the strong anisotropy already

TABLE I. Fitting parameters for the rates shown in Fig. 3(b), using the Keldysh fit formula $R(E)=P\left[E-E_{\mathrm{th}}\right]^{a}$.

\begin{tabular}{lccc}
\hline \hline & $E_{t h}(\mathrm{eV})$ & $a$ & $P\left(\mathrm{~s}^{-1} \mathrm{eV}^{-a}\right)$ \\
\hline This work & 1.8 & 5.8 & $3.5 \times 10^{10}$ \\
Ref. 11 & 1.89 & 5.2 & $1.4 \times 10^{11}$ \\
Ref. 7 & 1.73 & 7.8 & $4.57 \times 10^{10}$ \\
Ref. 10 & 2.1 & 4.0 & $2 \times 10^{12}$ \\
\hline \hline
\end{tabular}

noted: carriers with the same impacting energy but different wave vectors can have widely varying rates. However, this anisotropy diminishes at higher energies, due to the greater ease with which $\mathbf{k}$ and energy conservation restrictions can be satisfied. Moreover, it is of interest to show an "isotropic" II rate, that depends only on the impacting electron energy $E$ [solid line in Fig. 3(a)]:

$$
R(E)=\frac{\sum_{n_{1}} \int d^{3} \mathbf{k}_{1} \delta\left[E_{\mathbf{k}_{1}}^{n_{1}}-E\right] r\left(n_{1}, \mathbf{k}_{1}\right)}{\sum_{n_{1}} \int d^{3} \mathbf{k}_{1} \delta\left[E_{\mathbf{k}_{1}}^{n_{1}}-E\right]} .
$$

The physical reason to obtain an isotropic energy dependent rather than a wave-vector dependent rate is that in most common technological devices in which high electric fields usually are present, carriers are scattered by phonons, so as to reach similar energies, but largely different wave vectors.

Further, our $E$-dependent rate has been fitted using a "Keldysh-type" formula [dashed line in Fig. 3(a)], $R(E)$ $=P\left[E-E_{\mathrm{th}}\right]^{a}$ where $E_{\mathrm{th}}$ is the "isotropic" threshold energy, i.e., the minimum energy at which the carrier is able to excite an $e^{-}$in the valence band and, therefore, to initiate II. Here, $P, a$, and $E_{\mathrm{th}}$ have been treated as fitting parameters. An optimized linear regression procedure yields a fitted value $E_{\mathrm{th}}^{\mathrm{fit}} \sim 1.8 \mathrm{eV}$, whereas our simulations indicate no ionization events for impacting energies lower than $E_{\mathrm{th}}^{\text {calc }} \sim 1.86 \mathrm{eV}$ (considered as our "real" value for $E_{\mathrm{th}}$ ). The excellent agreement between $E_{\mathrm{th}}^{\mathrm{fit}}$ and $E_{\mathrm{th}}^{\text {calc }}$ provides confidence in the numerical fit. As a result, the threshold is slightly higher than

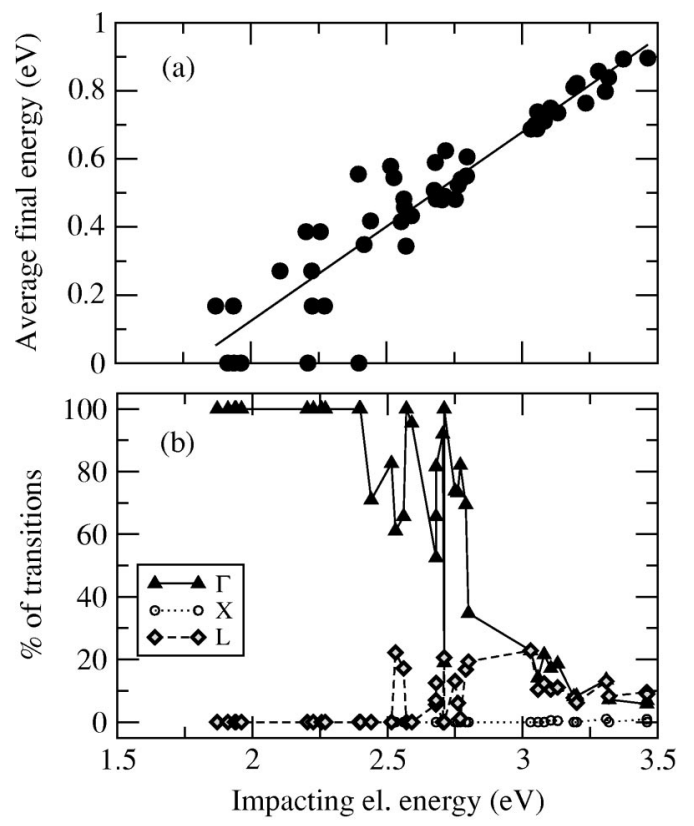

FIG. 4. (a) Average final $e^{-}$energy (filled circles) vs impacting $e^{-}$energy (in $\mathrm{eV}$ ) referred to the conduction band minimum (zero of energy). The solid line shows the best linear fit. (b) Percentage of transitions that involve final states at the symmetry point $[\Gamma$ (triangles), $L$ (diamonds), and $X$ (circles)] valleys vs impacting $e^{-}$ energies (in $\mathrm{eV}$ ). 
the energy gap and in good agreement with the relation $E_{\text {th }}$ $=3 / 2 E_{\text {gap }}$ obtained from a parabolic band structure with constant effective masses. ${ }^{1}$ However, we believe this agreement to be fortuitous, since we have shown previously the importance of a careful treatment of band anisotropy. Moreover, it is noted that the value of $E_{\text {th }}$ is exact within the uncertainty given by $\Delta_{E}$. From the fit, we obtained $P=3.5$ $\times 10^{10} \mathrm{~s}^{-1} \mathrm{eV}^{-a}$ and $a \sim 5.8$; the high value of $a$ reveals the "soft" character of the GaAs threshold.

In Table I and Fig. 3 (b) we compare the result of our fit with other Keldysh-type fits available from MC (Ref. 7) or EPP (Refs. 10,11) methods. Our ab initio results are in overall good agreement-especially at low energies-with two of the previous works. ${ }^{11,7}$ While the threshold energies are within a very similar range, there are some differences in the $P$ and $a$ values; these can be ascribed mainly to the details of band structures and numerical methods employed for evaluating the rates (e.g., many-fold integration schemes).

While it would be important to compare our results with experiment, the II rate is not directly comparable. Rather, the quantity commonly measured is the electron ionization coefficient $\alpha(F)$ (in $\mathrm{cm}^{-1}$ ) as a function of the applied field $F$. This coefficient is related to the inverse of the mean distance traveled by carriers prior to II; by contrast, our calculated rates (when summed with the phonon rates and normalized) give the probability (in $\mathrm{s}^{-1}$ ) of an II event as a function of the carrier's energy. Therefore, the calculated II rates and the measured II coefficient are not easily related. For the experiments, we refer to Bulman et al. ${ }^{22}$, who measured $\alpha(F)$ in
(100) GaAs in a large number of different $p^{+} n$ structures using avalanche noise and photocurrent multiplication: their electron ionization threshold is $1.7 \mathrm{eV}$. Therefore, the agreement with our value of $\sim 1.8 \mathrm{eV}$ supports the reliability of our procedure.

Finally, we offer some information about the distribution of final states that is of great interest for transport simulations. In Fig. 4(a), we plot the average final electron energy vs impacting electron energy along with the best linear fit. Similar to the rates themselves, the scattering of points off the straight line is evident in the low energy region. The average final electron energy is not necessarily equal, even for primary impacting electrons having the same initial energy, because of the strong restrictions imposed by $\mathbf{k}$ conservation. On the other hand, as the impacting energy increases the linear fit improves significantly. Moreover, we plot in Fig. 4(b) the percentage of transitions that involve one or both final states in the symmetry point valleys $(L, \Gamma, X)$ vs the impacting electron energies. As expected, at low energy, most of the transitions (even $100 \%$ at some energies) involve one or even both final states in the $\Gamma$ valley. However, as the impacting electron energy increases, the other valleys (especially the $L$ valley) become accessible and the final states are generally more spread out over the BZ.

Work at Northwestern University was supported by the NSF (through the N.U. Materials Research Center). We thank Dr. Wolfgang Mannstadt for early discussions and Dr. Ilmun $\mathrm{Ju}$ for providing helpful information.
*Electronic mail: silvia.picozzi@aquila.infn.it

${ }^{1}$ P.T. Landsberg, Recombination in Semiconductors (Cambridge University Press, Cambridge, 1991).

${ }^{2}$ K.F. Brennan, The Physics of Semiconductors (Cambridge University Press, Cambridge, 1999).

${ }^{3}$ D.M. Bylander and L. Kleinman, Phys. Rev. B 41, 7868 (1990).

${ }^{4}$ A. Seidl, A. Gorling, P. Vogl, J.A. Majewski, and M. Levy, Phys. Rev. B 53, 3764 (1996).

${ }^{5}$ W. Kohn and L.J. Sham, Phys. Rev. A 140, A1113 (1965); P. Hohenberg and W. Kohn, Phys. Rev. B 136, B864 (1964).

${ }^{6}$ J. Bude and K. Hess, J. Appl. Phys. 72, 3554 (1992).

${ }^{7}$ H.K. Jung, K. Taniguchi, and C. Hamaguchi, J. Appl. Phys. 79, 2473 (1996).

${ }^{8}$ Y. Wang and K. Brennan, J. Appl. Phys. 71, 2736 (1992).

${ }^{9}$ N. Sano and A. Yoshii, Phys. Rev. B 45, 4171 (1992).

${ }^{10}$ M. Stobbe, R. Redmer, and W. Schattke, Phys. Rev. B 49, 4494 (1994).

${ }^{11}$ D. Harrison, R.A. Abram, and S. Brand, J. Appl. Phys. 85, 8186 (1999).

${ }^{12}$ G. Cappellini, R. Del Sole, L. Reining, and F. Bechstedt, Phys. Rev. B 47, 9892 (1993).

${ }^{13}$ R. Asahi, W. Mannstadt, and A.J. Freeman, Phys. Rev. B 59, 7486 (1999).
${ }^{14}$ C.B. Geller, W. Wolf, S. Picozzi, A. Continenza, R. Asahi, W. Mannstadt, A.J. Freeman, and E. Wimmer, Appl. Phys. Lett. 78, 368 (2001).

${ }^{15}$ E. Wimmer, H. Krakauer, M. Weinert, and A.J. Freeman, Phys. Rev. B 24, 864 (1981).

${ }^{16}$ H.J. Monkhorst and J.D. Pack, Phys. Rev. B 13, 5188 (1976).

${ }^{17}$ S. Massidda, M. Posternak, and A. Baldereschi, Phys. Rev. B 48, 5058 (1993).

${ }^{18}$ The effect of spin-orbit coupling in GaAs was investigated in D. Harrison, R.A. Abram, and S. Brand, J. Appl. Phys. 85, 8178 (1999), showing that the overall effect was relatively small. The effect of Umklapp terms was investigated in M. V. Fischetti, N. Sano, S. E. Laux, and K. Natori, IEEE Trans. Semicond. Technol.-Modeling and Simulations (to be published) resulting in unaltered rates in the threshold region and higher rates for impacting energies higher than $3 \mathrm{eV}$. http.//www.ieee.org/ products/online/journal/tcad/accepted/fischetti-feb97

${ }^{19}$ R. Redmer, J.R. Madureira, N. Fitzer, S.M. Goodnick, W. Schattke, and E. Scholl, J. Appl. Phys. 87, 781 (2000).

${ }^{20}$ T.P. Humphreys and G.P. Srivastava, Phys. Solid State 112, 581 (1982).

${ }^{21}$ M.L. Cohen and T.K. Bergstrasser, Phys. Rev. 141, 789 (1966).

${ }^{22}$ G. E. Bulman, V. M. Robbins, G. E. Stillman, IEEE Trans. Electron Devices ED-32, 2454 (1986). 